\title{
ON THE SIZE OF THE NON-COINCIDENCE SET OF PARABOLIC OBSTACLE PROBLEMS WITH APPLICATIONS TO AMERICAN OPTION PRICING
}

\author{
TEITUR ARNARSON and JONATAN ERIKSSON*
}

\begin{abstract}
The following paper is devoted to the study of the positivity set $U=\{\mathscr{L} \phi>0\}$ arising in parabolic obstacle problems. It is shown that $U$ is contained in the non-coincidence set with a positive distance between the boundaries uniformly in the spatial variable if the boundary of $U$ satisfies an interior $C^{1}$-Dini condition in the space variable and a Lipschitz condition in the time variable. We apply our results to American option pricing and we thus show that the positivity set is strictly contained in the continuation region, which means that the option should not be exercised in $U$ or on the boundary of $U$.
\end{abstract}

\section{Introduction}

The problem of pricing American contingent claims is the same as finding a sufficiently regular solution to the obstacle problem

$$
\begin{aligned}
& \mathscr{L} f \leq 0, \\
& f \geq \phi, \\
& (f-\phi)(\mathscr{L} f)=0
\end{aligned}
$$

in some open set $\Omega \subset \mathrm{R}^{n+1}$ with some appropriate boundary conditions on the parabolic boundary of $\Omega$. Here $\phi=\phi(x, t)$ is the pay-off function (the obstacle) and $\mathscr{L}$ is the differential operator

$$
\mathscr{L} f=\sum_{i, j=1}^{n} a_{i j} f_{i j}+\sum_{i=1}^{n} b_{i} f_{i}+c f-f_{t} .
$$

The non-coincidence set $\mathscr{C}=\{f>\phi\}$ is in option pricing called the continuation region, and in this set the option price is a solution to $\mathscr{L} f=0$. On the parabolic boundary of $\mathscr{C}$ we have continuity so that $f=\phi$ and on the lateral boundary we have the so-called principle of smooth fit $\nabla_{x}(f-\phi)=0$. The

\footnotetext{
* Teitur Arnarson was supported by STINT.

Received October 5, 2005.
} 
positivity set is defined as the set where the obstacle is a strict subsolution to $\mathscr{L}$, that is $U=\{\mathscr{L} \phi>0\}$, and it is always a subset of the continuation region. In the present paper we show that this inclusion must be strict in the sense that there is $\delta(t)>0$ such that the distance $\operatorname{dist}\left(\partial \mathscr{C}_{t}, \partial U_{t}\right) \geq \delta(t)$, where $\mathscr{C}_{t}=\{x \mid(x, t) \in \mathscr{C}\}$ and $U_{t}=\{x \mid(x, t) \in U\}$, and $\delta(t)$ depends only on $\mathscr{L}, \phi, n$ and $\Omega$.

\section{Notation}

We will use the following notation throughout the paper.

$\begin{array}{ll}K, K_{0}, K_{n}, \ldots & \text { generic constants } \\ D & \text { A domain in } \mathrm{R}^{n} \\ Q_{T} & \text { A cylindrical domain }\left\{(x, t) \in \mathrm{R}^{n+1} \mid x \in D, 0 \leq t \leq T\right\} \\ \Omega & \text { A domain in } \mathrm{R}^{n+1} \\ \left(x^{\prime}, t\right) & \text { A point }\left(x^{\prime}, t\right)=\left(x_{2}, x_{3}, \ldots, x_{n}, t\right) \in \mathrm{R}^{n-1} \times \mathrm{R} \\ (x, t) & \text { A point }(x, t)=\left(x_{1}, x_{2}, \ldots, x_{n}, t\right) \in \mathrm{R}^{n+1} \\ \mathrm{R}_{+}^{n} & \left\{x \in \mathrm{R}^{n} \mid x_{1}>0\right\} \\ B_{r}(x) & \left\{y \in \mathrm{R}^{n}|| y-x \mid<r\right\} \\ B_{r}^{+}(x) & B_{r}(x) \cap \mathrm{R}_{+}^{n} \\ B_{r}, B_{r}^{+} & B_{r}(0), B_{r}^{+}(0) \\ C_{r}(x, t), C_{r}^{+}(x, t) & B_{r}(x) \times\left(t-r^{2}, t+r^{2}\right), B_{r}^{+}(x) \times\left(t-r^{2}, t+r^{2}\right) \\ C_{r}, C_{r}^{+} & C_{r}(0,0), C_{r}^{+}(0,0) \\ A(x, t) & \text { A } n \times n \text { matrix-valued function with elements } a_{i j}(x, t) \\ \tilde{A} & \text { The }(n-1) \times(n-1) \text { matrix-valued function obtained by } \\ & \text { removing the first row and column from } A .\end{array}$

\section{Assumptions, technical tools and known results}

In this section we make all the necessary assumptions and we gather some technical tools and known results that will be useful in proving the main result.

Definition 3.1. A modulus of continuity $\alpha$ is called a Dini modulus of continuity if $\int_{0^{+}} \frac{\alpha(r)}{r} d r<\infty$ and a function $h$ is called Dini continuous if $h$ has a Dini modulus of continuity. We define the function spaces $C_{D, \alpha}(\Omega)$ which consists of all functions $h: \Omega \rightarrow \mathrm{R}$ such that $h$ is Dini continuous with modulus of continuity $\alpha$, and $C_{D, \alpha}^{k, l}(\Omega)$ as the space of functions $h: \Omega \rightarrow \mathrm{R}$ such that $D_{x}^{m_{1}} h \in C_{D, \alpha}(\Omega)$ and $D_{t}^{m_{2}} h \in C_{D, \alpha}(\Omega)$ for every multi-index $m_{1}$ and every positive integer $m_{2}$ such that $\left|m_{1}\right| \leq k$ and $m_{2} \leq l$.

Consider the operator $\mathscr{L} f=\sum_{i, j=1}^{n} a_{i j}(x, t) f_{i j}+\sum_{i=1}^{n} b_{i}(x, t) f_{i}$ $+c(x, t) f-f_{t}$ mentioned in Section 1. We make the following assumptions regarding the regularity of the coefficients of the operator. 
Assumption 3.2. The lower order coefficients satisfy $b_{i}, c \in L_{\mathrm{loc}}^{\infty}\left(\mathrm{R}^{n+1}\right)$ and the real symmetric matrix $A(x, t)$ is uniformly positive definite uniformly in $x$ and $t$ and the $L_{\text {loc }}^{\infty}$-norm of $A$ is finite and $A$ is $C_{D}^{1,0}$ on compact subsets in the parabolic norm. That is we assume that $\left|a_{i j}(x, t)-a_{i j}(y, s)\right| \leq \alpha(\mid(x, t)-$ $(y, s) \mid)$ and $\left|\frac{\partial a_{i j}(x, t)}{\partial x_{i}}-\frac{\partial a_{i j}(y, s)}{\partial x_{i}}\right| \leq \alpha^{\prime}(|(x, t)-(y, s)|)$ where $\alpha$ and $\alpha^{\prime}$ are two Dini moduli of continuity and the norm $|(x, t)|$ on the right hand side is the parabolic norm.

Under these assumptions it is shown in [5] that a weak solution of $\mathscr{L} u=$ $g$ has a continuous and locally bounded gradient for any $g \in L_{\text {loc }}^{\infty}\left(\mathrm{R}^{n+1}\right)$. Notice that since $A$ has continuous partial derivatives we may write $\mathscr{L}$ in divergence form instead. In this case $\mathscr{L} f=\nabla \cdot\left(A(x, t)(\nabla f(x, t))^{T}\right)+$ $B(x, t) \cdot(\nabla f(x, t))^{T}+c f-\frac{\partial f}{\partial t}$ where the $i$ th component of the vector $B$ is $b_{i}(x, t)-\sum_{j=1}^{n} \frac{\partial a_{i j}(x, t)}{\partial x_{j}}$. This will come in handy later, when we change variables to flatten the boundary locally, because in the new coordinates $(y, s)$ the coefficient $A_{y}(y, s)$ of the operator $\mathscr{L}$ need not be differentiable in $y$.

\subsection{Local representations}

Given a set $\Omega$ and a point $\left(x^{0}, t^{0}\right)$ on its boundary, we say that $h: \mathrm{R}^{n-1} \times \mathrm{R} \rightarrow \mathrm{R}$ is a local representation of $\Omega$ at $\left(x^{0}, t^{0}\right)$ if there are constants $\delta>0$ and $K_{1}>0$ such that after suitable rotation

$$
\Omega \cap C_{\delta}\left(x^{0}, t^{0}\right)=\left\{(x, t) \in \mathrm{R}^{n+1} \mid x_{1}>h\left(x^{\prime}, t\right)\right\} \cap C_{\delta}\left(x^{0}, t^{0}\right)
$$

and

$$
\left|h\left(x_{1}^{\prime}, t_{1}\right)-h\left(x_{2}^{\prime}, t_{2}\right)\right| \leq K_{1}\left(\left|x_{1}^{\prime}-x_{2}^{\prime}\right|+\left|t_{1}-t_{2}\right|^{1 / 2}\right) \quad \text { in } \quad C_{\delta}\left(x^{0}, t^{0}\right) .
$$

Note that if $h$ is local representation of $\Omega$ at $\left(x^{0}, t^{0}\right)$ then $\left(x^{0}, t^{0}\right)$ must be a lateral boundary point of $\Omega$. The boundary of $\Omega$ is said to be $C^{1}$-Dini at $\left(x^{0}, t^{0}\right)$ if there is a Dini modulus of continuity $\omega$ such that the local representation $h$ of $\partial \Omega$ satisfies

$$
\begin{aligned}
\left|\nabla_{x^{\prime}} h\left(x_{1}^{\prime}, t_{1}\right)-\nabla_{x^{\prime}} h\left(x_{2}^{\prime}, t_{2}\right)\right| & \leq \omega\left(\left|x_{1}^{\prime}-x_{2}^{\prime}\right|\right)+\omega\left(\left|t_{1}-t_{2}\right|^{1 / 2}\right) \\
\left|h\left(x^{\prime}, t_{1}\right)-h\left(x^{\prime}, t_{2}\right)\right| & \leq K_{2}\left|t_{1}-t_{2}\right| .
\end{aligned}
$$

(see for instance [3] for more details on local representations). A set $\Omega$ is said to have an interior $C^{1}$-Dini property at $\left(x^{0}, t^{0}\right)$ if there is a set $\Omega^{\prime} \subset \Omega$ such that $\partial \Omega \cap \partial \Omega^{\prime}=\left\{\left(x^{0}, t^{0}\right)\right\}$ and if $\partial \Omega^{\prime}$ is $C^{1}$-Dini at $\left(x^{0}, t^{0}\right)$ and it is simply said to have an interior $C^{1}$-Dini property if all boundary points of $\Omega$ has an interior $C^{1}$-Dini property. 


\subsection{Straightening out the boundary and a change of variables}

Assume that the set $\Omega$ is $C^{1}$-Dini at a lateral boundary point $\left(x^{0}, t^{0}\right)$. The boundary may now be straightened out with a $C^{1}$ change of variables. Namely if we put

$$
\left\{\begin{aligned}
y_{1} & =x_{1}-h\left(x^{\prime}, t\right) \\
y_{2} & =x_{2}-x_{2}^{0} \\
& \vdots \\
y_{n}=x_{n}-x_{n}^{0} & \\
s & =t-t^{0}
\end{aligned}\right.
$$

then the boundary is flat in a neighborhood of $\left(x^{0}, t^{0}\right)$ in the new coordinate system $(y, s)$. That is, for some $\delta^{\prime}>0$ possibly smaller than $\delta$ there is $C_{\delta^{\prime}}$ such that in the new coordinate system $(y, s), \Omega \cap C_{\delta^{\prime}}=\left\{(y, s) \in \mathrm{R}^{n+1} \mid y_{1}>\right.$ $0\} \cap C_{\delta^{\prime}}$.

In view of this maneuvere the analysis of solutions to $\mathscr{L} f=g$ in a neighborhood of a boundary point $\left(x^{0}, t^{0}\right)$ may as well be carried out in the $(y, s)$ coordinates in the neighborhood $\Omega \cap C_{\delta^{\prime}}$ of $(0,0)$ with the boundary being $\left\{y_{1}=0\right\} \times\left(-\left(\delta^{\prime}\right)^{2},\left(\delta^{\prime}\right)^{2}\right)$ in a neighborhood of $(0,0)$. The change of coordinates affects the operator $\mathscr{L}$ as well and it will in general be much more complicated in the new coordinates. The explicit expression can be obtained as follows. Define a matrix function $A_{y}(y, s)$ and a vector function $B_{y}(y, s)$ by

$$
A_{y}(y, s)=A(x(y, s), t(s))+\left(\begin{array}{cc}
\nabla_{y^{\prime}} h \cdot\left(A \nabla_{y^{\prime}} h\right) & -\left(\nabla_{y^{\prime}} h\right) \tilde{A} \\
-\tilde{A}\left(\nabla_{y^{\prime}} h\right)^{T} & 0
\end{array}\right)
$$

and $B_{y}(y, s)=\left(B_{1}-B \cdot \nabla_{y} h+\frac{\partial h}{\partial s}, B^{\prime}\right)$ where $B=\left(B_{1}, B^{\prime}\right)$. Here $X^{T}$ denotes the transpose of the matrix $X$ and we substitute the expression for $y$ according to (3.3) instead of $x$. The operator expressed in the coordinates $(y, s)$ becomes

$$
\begin{aligned}
\mathscr{L}_{y} F(y, s)=\nabla \cdot\left(A_{y}(y, s)\right. & \cdot \nabla F(y, s)) \\
& +B_{y}(y, s) \cdot \nabla F(y, s)+C_{y}(y, s) F-\frac{\partial F}{\partial s},
\end{aligned}
$$

where $\nabla=\nabla_{y}$ is the gradient with respect to the y-variables. Then if $f$ is a solution to $\mathscr{L} f=g$ in $\Omega \cap C_{\delta}\left(x^{0}, t^{0}\right)$ then $F(y, s)=f\left(y_{1}+h\left(y^{\prime}, s\right), y^{\prime}, s\right)$ is a solution to $\mathscr{L}_{y} F(y, s)=G(y, s)$ in $\Omega \cap C_{\delta^{\prime}}$ in the $(y, s)$ coordinates.

Proposition 3.3. The operator $\mathscr{L}_{y}$ is well-defined and the coefficients satisfy $\left(B_{y}\right)_{i}, C \in L^{\infty}\left(\Omega \cap C_{\delta^{\prime}}\right)$ and the real matrix $A_{y}(y, s)$ is uniformly positive 
definite uniformly in $y$ and $s$ and the $L^{\infty}\left(\Omega \cap C_{\delta^{\prime}}\right)$-norm of $A_{y}$ is finite and $A_{y}$ is in $C_{D}\left(\Omega \cap C_{\delta^{\prime}}\right)$.

Proof. We need to verify that $B_{y}$ is bounded and that $A_{y}$ is Dini continuous in $\Omega \cap C_{\delta^{\prime}}$ and since all components except for the first in the vector $b$ is unchanged we only need to investigate $\left(B_{y}\right)_{1}$. From (3.2) it follows that

$$
\left|\frac{\partial h(y, s)}{\partial s}\right| \leq K_{2} \text {. }
$$

It follows that $\left(B_{y}\right)_{1}$ is bounded in $\Omega \cap C_{\delta^{\prime}}$. Next we verify that $A_{y}$ is Dini continuous in $\Omega \cap C_{\delta^{\prime}}$. The first term on the right hand side of Equation 3.4 is $A\left(\left(y_{1}+h\left(y^{\prime}, s\right), y^{\prime}+\left(x^{0}\right)^{\prime}\right), s+t^{0}\right)$ and a direct calculation shows that $2 \sqrt{2} K_{1} \alpha$ is a Dini modulus of continuity for this term in the variables $(y, s)$. The second term involves the product of two Dini-continuous functions and we can find another constant $K_{3}$ such that $2 \sqrt{2} K_{1} \alpha+K_{3}(\alpha+\omega)$ is a Dini modulus of continuity for $A_{y}$ and the constant $K_{3}$ depends only on $K_{1}$, $\sup _{\Omega \cap C_{\delta^{\prime}}}|\nabla h|$ and $\sup _{\Omega \cap C_{\delta^{\prime}}}|A|$.

\section{Local existence and regularity of solutions to variational inequalities}

In this section we give some existence and regularity results of local character for the obstacle problem (1.1)-(1.3) from Section 1. The proof of existence and regularity is standard, and by combining results on continuity of the gradient of weak solutions from [5] with results from [6] on existence of solution with weak derivatives to Cauchy-Dirichlet problems when the top-order coefficient is only continuous, we can use the method of penalization (see for instance [2]) to obtain a unique solution with a locally uniformly continuous spatial gradient and weak second order derivative in space and weak first order derivative in time.

THEOREM 4.1. Assume that the coefficients of the operator $\mathscr{L}$ satisfies Assumption (3.2). Then for any cylinder bounded $Q_{T}$ and for any function $g \in C^{2,1}$ on $\overline{Q_{T}}$ such that $g \geq \phi$ and $g(x, 0)=\phi(x, 0)$ there is a unique solution of

$$
\begin{aligned}
& \mathscr{L} f \leq 0, \\
& f \geq \phi, \\
& (f-\phi)(\mathscr{L} f)=0, \\
& \left.f\right|_{\partial_{p} Q_{T}}=\left.g\right|_{\partial_{p} Q_{T}} .
\end{aligned}
$$

Moreover, the solution is in $W_{p}^{2,1}\left(Q_{T}\right)$ for any $1<p<\infty$ and $\nabla_{x} f$ is uniformly continuous on $\overline{Q_{T}}$. 
Proof. The idea is identical to the one in proof of Theorem 3.2 in [2] and we use the penalization method implemented there. That is we take a $C^{\infty}$ function $\beta_{\epsilon}(t)$ satisfying

$$
\begin{aligned}
& \beta_{\epsilon}^{\prime}(t) \geq 0, \\
& \beta_{\epsilon}(t) \rightarrow-\infty \quad \text { if } \quad t<0, \epsilon \rightarrow 0 \text {, } \\
& \beta_{\epsilon}(t) \rightarrow 0 \quad \text { if } \quad t \geq 0, \epsilon \rightarrow 0 \text {, } \\
& \beta_{\epsilon}(t) \leq C, \\
& \beta_{\epsilon}(0) \geq-C,
\end{aligned}
$$

where $C$ is a constant independent of $\epsilon$, and we study solutions to the penalized problem

$$
\begin{aligned}
\mathscr{L} f-\beta_{\epsilon}(f-\phi) & =0 \quad \text { in } \quad Q_{T}, \\
\left.f\right|_{\partial_{p} Q_{T}} & =\left.g\right|_{\partial_{p} Q_{T}} .
\end{aligned}
$$

We claim that there exists a solution $f_{\epsilon}$ to to Equation (4.5) with boundary value (4.6). If we put $\beta_{\epsilon, N}(t)=\max \left\{\min \left\{\beta_{\epsilon}(t), N\right\},-N\right\}$ it follows from Theorem 9.1, Ch. 4 in [6] that for each $q>1$ and for each $h \in L^{q}\left(Q_{T}\right)$ there is a $W_{q}^{2,1}\left(Q_{T}\right)$ solution $f=f_{\epsilon, N}$ to

$$
\begin{aligned}
\mathscr{L} f-\beta_{\epsilon, N}(h-\phi) & =0 \quad \text { in } \quad Q_{T}, \\
\left.f\right|_{\partial_{p}} Q_{T} & =\left.g\right|_{\partial_{p}} Q_{T} .
\end{aligned}
$$

Moreover, the solution satisfies $|f|_{W_{q}^{2,1}\left(Q_{T}\right)} \leq K$ for some constant $K$ independent of $h$. Since the right hand side is bounded for each $N$ it follows from [5] that $\nabla_{x} f_{\epsilon, N}$ is uniformly continuous on $\overline{Q_{T}}$ for each $N$ and each $\epsilon$. From a corollary to Theorem 9.1, Ch. 4 in [6] we know that $f$ is Hölder continuous, which means that $\beta_{\epsilon, N}(f-\phi)$ is Hölder continuous. We shall try to estimate this function. Therefore we put $\xi(x, t)=\beta_{\epsilon, N}(f-\phi)(x, t)$ and suppose that $\left(x_{0}, t_{0}\right)$ is the minimum point of $\xi$ and that $\mu=\xi\left(x_{0}, t_{0}\right)$ and $\mu \leq 0$, $\mu<\beta_{\epsilon}(0)$. If $\left(x_{0}, t_{0}\right) \in \partial_{p} Q_{T}$ then $\mu=\beta_{\epsilon, N}\left(g\left(x_{0}, t_{0}\right)-\phi\left(x_{0}, t_{0}\right)\right) \geq \beta_{\epsilon}(0)$. Hence $\left(x_{0}, t_{0}\right) \in Q_{T}$, and since $\beta_{\epsilon, N}(t)$ is monotone in $t, f-\phi$ must have a non-positive minimum at $\left(x_{0}, t_{0}\right)$. Hence $\mathscr{L}\left(f\left(x_{0}, t_{0}\right)-\phi\left(x_{0}, t_{0}\right)\right) \geq 0$ and it follows from Equation (4.7) that $\xi\left(x_{0}, t_{0}\right) \geq \mathscr{L} \phi\left(x_{0}, t_{0}\right) \geq-\tilde{C}$ for some constant $\tilde{C}$ independent of $N$ and $\epsilon$. Thus, $\left|\beta_{\epsilon, N}(f-\phi)\right| \leq \max \{C, \tilde{C}\}:=C_{1}$, which is independent of $N$ and $\epsilon$. Hence, for $N$ large enough we have that $\beta_{\epsilon, N}(f-\phi)=\beta_{\epsilon}(f-\phi)$ and it follows that for $N$ large enough $f=f_{\epsilon, N}$ is a solution to Equation (4.5) with boundary value (4.6) and which satisfies $|f|_{W_{q}^{2,1}\left(Q_{T}\right)} \leq C_{1}$. By weak compactness we can find a subsequence $\epsilon_{k}$ such that $\epsilon_{k} \rightarrow 0$ as $k \rightarrow \infty$ and $f_{\epsilon_{k}}$ converges weakly in $W_{q}^{2,1}\left(Q_{T}\right)$. Call the limit 
function $f$. Both $f_{\epsilon}$ and $\nabla_{x} f_{\epsilon}$ are uniformly continuous on $\bar{Q}_{T}$ and it follows that $f_{\epsilon}$ converges uniformly to $f$ on $\bar{Q}_{T}$ and that $\nabla_{x} f$ is uniformly continuous on $\bar{Q}_{T}$. Moreover, since $\left|\beta_{\epsilon}\left(f_{\epsilon}-\phi\right)\right| \leq C_{1}$ we deduce that $f \geq \phi$, and since $\beta_{\epsilon}\left(f_{\epsilon}-\phi\right) \rightarrow 0$ on $\{f>\phi\}$ the equation $\mathscr{L} f=0$ holds on $\{f>\phi\}$. By the definition of $\beta_{\epsilon}$ we can also conclude that $\lim \sup _{\epsilon \rightarrow 0} \beta_{\epsilon}\left(f_{\epsilon}-\phi\right) \leq 0$ and thus that $\mathscr{L} f \leq 0$. The function $f$ is the desired solution to the obstacle problem and $f \in W_{q}^{2,1}\left(Q_{T}\right)$ for any $q \in(1, \infty)$. Moreover, $\nabla_{x} f$ is uniformly continuous on $\bar{Q}_{T}$.

\section{A Hopf-type lemma for parabolic operators}

In this section we show that the interior ball condition needed in the proof of the classical Hopf boundary point lemma (see for instance [1]) is unnecessarily strong. In fact it is enough if the boundary is $C_{D}^{1}$ for the lemma to hold. The main argument uses Theorem 1.5.10 in [5] and the maximum principle.

Lemma 5.1. Assume that $u \geq 0$ is a weak solution to the equation $\mathscr{L} u=$ $h \leq 0$ and $u>0$ in the interior of $\Omega \cap C_{\delta}\left(x^{0}, t^{0}\right)$ and that $u\left(x^{0}, t^{0}\right)=0$. Assume also that $\Omega$ is $C_{D}^{1}$ at $\left(x^{0}, t^{0}\right)$. Let $n$ be the inward unit normal at $\left(x^{0}, t^{0}\right)$ for $\partial \Omega$. Then the following holds

$$
\frac{\partial u\left(x^{0}, t^{0}\right)}{\partial n} \geq C>0
$$

for some constant $C$.

Proof. In view of the flattening of the boundary described in the previous section we may assume that $\Omega \cap C_{\delta}=\left\{x_{1}>0\right\} \cap C_{\delta}$ for some $\delta>0$. Denote this set by $\Omega_{\delta}$. Let $\eta_{0}$ be as in Theorem 1.5.10 in [5] and take $\eta \leq \eta_{0}$ such that $\eta \leq \min \left(\eta_{0}, \delta\right)$. By a shift in time and space we can consider Theorem 1.5.10 in $I_{\eta}=B_{\eta / 2}\left(x_{0}\right) \times\left[-\eta^{2} / 2, \eta^{2} / 2\right]$ where $x_{0}=(\eta / 2,0,0, \ldots, 0)$. Let $0<K<\infty$ be a constant such that $K \inf _{\eta / 4 \leq\left|x-x_{0}\right| \leq 3 \eta / 4} u(x,-\eta / 2) \geq 1$. Such a constant can be found since $u$ is continuous and positive for $x$ in the compact set $\eta / 4 \leq\left|x-x_{0}\right| \leq 3 \eta / 4$. Take a $C_{0}^{\infty}\left(\mathrm{R}^{n}\right)$ function $\theta$ with the support of $\theta$ being a subset of $\left|x-x_{0}\right| \in(\eta / 4,3 \eta / 4)$ and such that $0 \leq \theta \leq 1$ and $\theta=1$ for $\left|x-x_{0}\right| \in(3 \eta / 8,5 \eta / 8)$ and put $\Theta_{\eta}(x, t)=\theta(x)$. Take $v$ to be the solution to

$$
\begin{aligned}
\mathscr{L} v & =K h \quad \text { in } \quad I_{\eta} \\
\left.v\right|_{\partial_{p} I_{\eta}} & =\left.\Theta\right|_{\partial_{p} I_{\eta}} .
\end{aligned}
$$

By the maximum principle the inequality $K u \geq v$ holds in $I_{\eta}$. Hence $K \frac{\partial u}{\partial x_{1}}(0,0)$ $=\liminf _{h \rightarrow 0} \frac{K u(h, 0, \ldots, 0,0)}{h} \geq \liminf _{h \rightarrow 0} \frac{v(h, 0, \ldots, 0,0)}{h}=\frac{\partial v}{\partial x_{1}}(0,0) \geq C>0$ 
according to Theorem 1.5.10 in [5] where $C$ is independent of $t$ and $x$. Thus it follows that $\frac{\partial u(0,0)}{\partial x_{1}} \geq C / K$ which means that for some constant $C^{\prime}(K)$ we have $\frac{\partial u\left(x^{0}, t^{0}\right)}{\partial n} \geq C^{\prime}$ in the original coordinate system.

\section{Uniform distance between the continuation region and the positivity set}

The positivity set $U=\{\mathscr{L} \phi>0\}$ is a subset of the continuation region, but can the boundaries coincide? Given a pay-off function $\phi \in C_{D}^{2,1}(\Omega)$ the following theorem holds.

TheOREm 6.1. Assume, in addition to Assumption 3.2, that the coefficients $b_{i}$ and c all are in $C_{D}^{0,0}(\Omega)$. Then if the lateral part of $\partial U$ is $C_{D}^{1}$ at each point, there is $\delta(t)>0$ depending on $\mathscr{L}, \phi, n$ and $Q$ such that $\operatorname{dist}\left(\partial U_{t}, \partial \mathscr{C}_{t}\right) \geq \delta(t)$ for each $t \in(0, T]$.

Proof. Suppose that the theorem is false. Then there exist sequences of coefficients $a_{i j}^{m}, b_{i}^{m}, c^{m}$ satisfying Assumption 3.2 uniformly in $m$ and such that $b_{i}$ and $c$ all are in $C_{D}^{0,0}(\Omega)$ uniformly in $m$, a sequence of solutions $f^{m}$ to $\mathscr{L}_{m} f^{m}=0$ in the set $\mathscr{C}_{m}=\left\{f^{m}>\phi\right\}$ such that $\operatorname{dist}\left(\partial U_{t}^{m}, \partial \mathscr{C}_{t}^{m}\right) \leq \frac{1}{m}$. Moreover, each $U^{m}$ has a local representation at each point that is $C_{D}^{1}$ uniformly in $m$, and for each $m, f^{m}$ is a $W_{q}^{2,1}$-solution to

$$
\begin{aligned}
& \mathscr{L}_{m} f^{m} \leq 0, \\
& f^{m} \geq \phi, \\
& \left(f^{m}-\phi\right)\left(\mathscr{L}_{m} f^{m}\right)=0 .
\end{aligned}
$$

On each compact $K \subset \Omega$, the families $a_{i j}^{m}, b_{i}^{m}, c^{m}$ are equicontinuous so by Arzela-Ascoli there exist subsequences that converge uniformly to some $a_{i j}^{0}, b_{i}^{0}, c^{0}$ satisfying the Dini conditions above on $K$. Thus, the subsequence $\mathscr{L}_{m} \phi \rightarrow \mathscr{L}_{0} \phi$ uniformly and $U^{m} \rightarrow U$. Moreover, $f^{m}$ is locally uniformly bounded and since the coefficients $A^{m}, B^{m}$ and $C^{m}$ satisfies Assumption 3.2 uniformly in $m$ and also the extra continuity condition of the theorem, the spatial gradient of $f^{m}$ is uniformly continuous on $K$ uniformly in $m$. Thus by the Arzela-Ascoli theorem we may extract a subsequence from $f_{m}$ such that this sequence converges uniformly on $K$ to a function $f^{0} \in W_{q}^{2,1}(\Omega)$ for any $1 \leq q<\infty$ and such that $\nabla f^{m} \rightarrow \nabla f^{0}$ weakly in $L^{q}(K)$. Moreover, by Sobolev embedding $\nabla f^{0}$ is uniformly continuous on $K$. We need to prove that $f^{0}$ is a solution to the obstacle problem

$$
\begin{aligned}
& \mathscr{L}_{0} f^{0} \leq 0, \\
& f^{0} \geq \phi, \\
& \left(f^{0}-\phi\right)\left(\mathscr{L}_{0} f^{0}\right)=0 .
\end{aligned}
$$


Since the convergence is uniform it is evident that $f^{0} \geq \phi$ so we proceed by showing that $f^{0}$ satisfies the equation $\mathscr{L}_{0} f^{0}=0$ in the set $\mathscr{C}:=\left\{f^{0}>\phi\right\}$. Let $\left(x_{0}, t_{0}\right) \in\left\{f^{0}>\phi\right\}$. By the maximum principle we can find $r>0$ such that $f^{0}-\phi>\frac{1}{2}\left(f^{0}\left(\left(x_{0}, t_{0}\right)-\phi\left(x_{0}, t_{0}\right)\right)\right.$ in $C_{r}\left(x_{0}, t_{0}\right)$. By uniform convergence we can find an $M>0$ such that if $m>M$ then $f^{m}-\phi>f^{0}-\phi-\frac{1}{4}\left(f^{0}\left(x_{0}, t_{0}\right)-\right.$ $\left.\phi\left(x_{0}, t_{0}\right)\right)>0$ for all $(x, t) \in C_{r}\left(x_{0}, t_{0}\right)$. Hence $C_{r}\left(x_{0}, t_{0}\right) \subset\left\{f^{m}>\phi\right\}$ if $m>$ $M$ so on $C_{r}\left(x_{0}, t_{0}\right)$ we may take the limit in the equation to obtain $\mathscr{L}_{0} f^{0}=0$ on $C_{r}\left(x_{0}, t_{0}\right)$. Thus $f^{0}$ solves the equation $\mathscr{L}_{0} f^{0}=0$ on $\left\{f^{0}>\phi\right\}$. Next we take a smooth positive test function $\psi$ with compact support in $K$ and integrate $\int_{K}\left(\mathscr{L}_{m} f^{m}\right) \psi d x d t \leq 0$. By weak convergence and partial integration we can proceed to the limit to obtain $\int_{K}\left(\mathscr{L}_{0} f^{0}\right) \psi d x d t \leq 0$. Thus $f^{0}$ is the desired solution to the obstacle problem and the smooth fit condition $\nabla_{x} f^{0}=\nabla_{x} \phi$ is valid on $\partial\left\{f^{0}>\phi\right\} \cap K$. To finish the proof we observe that the sets $U$ and $\mathscr{C}$ must have at least one common boundary point. Take such a point and call it $\left(x_{0}, t_{0}\right)$. At this point both $f^{0}=\phi$ and $\nabla\left(f^{0}-\phi\right)=0$ and since $U \subset \mathscr{C}$ this contradicts the Hopf boundary point lemma from Section 5. Hence, the sequence above cannot exist and there has to be a uniform distance for each $t$ between $\partial U$ and $\partial \mathscr{C}$.

\section{Applications to mathematical finance}

Arbitrage valuation of American options in the Black-Scholes framework give rise to the obstacle problem (1.1)-(1.3) (see [7] chapter 8 for an introduction on American option valuation). In this setting the function $f$ is the option price and $\phi$ is the payoff of the option at the expiration date $T$ and the non-coincidence set is called the continuation region, since the option is not optimally exercised in this region.

An American option is a contract between two parties which gives the holder the following rights: At any time $\tau$ before the maturity time $T$ the holder may exercise the option, receiving the stochastic amount $\phi\left(S_{\tau}, \tau\right)$ from the seller. The seller has the obligation to pay $\phi\left(S_{\tau}, \tau\right)$ if the holder wants to exercise the option.

The stochastic process $S_{t}$ describes the time-evolution of the stock price and thus, $S_{\tau}$ is the stock price at time $\tau$. Two important questions in mathematical finance are what the value of such a contact is and when to exercise the option optimally. It is not obvious that it is optimal to exercise the option prior to the maturity time $T$. For instance if $\phi(s)=(s-K)^{+}$, i.e. $\phi$ is the pay-off of an American call option and if there is no dividends payed and if the volatility of the stock behaves "well" one should not exercise the option early (see Merton [4]).

The problem of pricing the option can either be approached from a stochastic 
point of view, by solving the optimal stopping problem which gives the price and the optimal exercise time, or by solving an obstacle problem and determine the non-coincidence set. Either of the approaches are in general difficult and one often has to rely on numerical results. However, we can say when not to exercise the option by studying the positivity set of the pay-off function. This set is contained in the continuation region and since the boundaries never coincide (under suitable regularity assumptions) we know also that when crossing the boundary of the positivity set one should hold the option at least a "little longer".

The financial model that we use is Black-Scholes model and it looks as follows: We assume that the stock-price process is an $n$-dimensional Itô-process $S_{t}=\left(S_{t}^{1}, \ldots S_{t}^{n}\right)$ driven by an $n$-dimensional Brownian motion $W_{t}$. The volatility $\sigma(s, t):=(\sigma(s, t))_{i j}$ and the risk-free rate of return $c(s, t)$ are deterministic matrix and real valued functions of current stock-price and time respectively. The drift of the underlying stock-price process under the risk-neutral measure will be denoted by $B(s, t):=\left(b^{1}(s, t), \ldots, b^{n}(s, t)\right)$ and is a vector valued function of current stock-price and time only. Notice that one usually has $B(s, t)=c(s, t) s$ under this measure but by being a little more general we allow for dividends (e.g. that $B(s, t)=(c(s, t)-\delta(s, t)) s$ for some dividend function $\delta(s, t)$ ). Thus we assume that $\sigma: \mathbf{R}^{n} \times \mathbf{R} \rightarrow \mathbf{R}^{n \times n}$ and $B: \mathbf{R}^{n} \times \mathbf{R} \rightarrow \mathbf{R}^{n}$ and $c: \mathbf{R}^{n} \times \mathbf{R} \rightarrow \mathbf{R}$, and to ensure uniqueness and existence of the process $S_{t}$ as a solution to a stochastic differential equation we also assume that $\left\|B\left(s_{1}, t\right)-B\left(s_{2}, t\right)\right\| \leq K\left\|s_{1}-s_{2}\right\|$, that $\left\|\sigma\left(s_{1}, t\right)-\sigma\left(s_{2}, t\right)\right\| \leq$ $K\left\|s_{1}-s_{2}\right\|$ and that $\|\sigma(s, t)\|+\|B(s, t)\| \leq K(1+\|s\|)$. We assume that the $i$ th asset $S_{t}^{i}$ is a solution to

$$
d S_{t}^{i}=b^{i}\left(S_{t}, t\right) d t+S_{t}^{i} \sum_{j=1}^{n} \sigma_{i j}\left(S_{t}, t\right) d W_{t}^{j}
$$

which by the assumptions we know is possible. The money-market (i.e. the bank-account) is given by

$$
B_{t}=B_{0} e^{\int_{0}^{t} c\left(S_{u}, u\right) d u} .
$$

Then the parabolic Black-Scholes operator associated with this process (applied to a function $f: \mathrm{R}^{n} \times \mathrm{R} \rightarrow \mathrm{R}$ ) is defined by

$$
\mathscr{L} f=\frac{1}{2} \sum_{i, j=1}^{n} s_{i} s_{j}\left(\sigma(s, t) \sigma^{T}(s, t)\right)_{i j} \frac{\partial^{2} f}{\partial s_{i} \partial s_{j}}+\sum_{i=1}^{n} b^{i}(s, t) \frac{\partial f}{\partial s_{i}}-c(s, t) f+\frac{\partial f}{\partial t}
$$

and the option price $f$ is a $C^{1}$-solution to the obstacle problem (1.1)-(1.3) with terminal condition $f(s, T)=\phi(s, T)$ (notice the changed direction of 
time). In the continuation region $\mathscr{C}=\{(s, t) ; f(s, t)>\phi(s, t)\}$ the equation $\mathscr{L} f=0$ is satisfied and the first optimal time to exercise the option is the first time the process $\left(S_{t}, t\right)$ hits the boundary of $\mathscr{C}$ (see [8] for a nice treatment of option pricing by variational inequalities). Generally closed formulas for solutions to problems of this type are not known and one usually has to rely on numerical results. By recalling the results from previous section we can at least say that if $\phi$ and if the coefficients of the Black-Scholes operator are sufficiently smooth, then it is not optimal to exercise the option when $\left(S_{t}, t\right)$ hits the boundary of the positivity set $U$ and we should in fact continue to hold the option a little longer. That is, the boundary of the positivity set can be used as an upper (or lower) bound of the free boundary. To illustrate our point we show in the graphs below how the boundaries of the sets $\mathscr{C}$ and $U$ are situated in some particular cases. In these calculations the model used for the stock-price is one-dimensional geometric Brownian motion (so that $\sigma$ is constant) and the pay-off functions used are $\phi(s, t)=\max \{0, K-s\}$, $\phi(s, t)=\max \{0, K-(1-t / 2) s\}$ and $\phi(s, t)=(K-s)^{2}+t^{2}$ respectively.
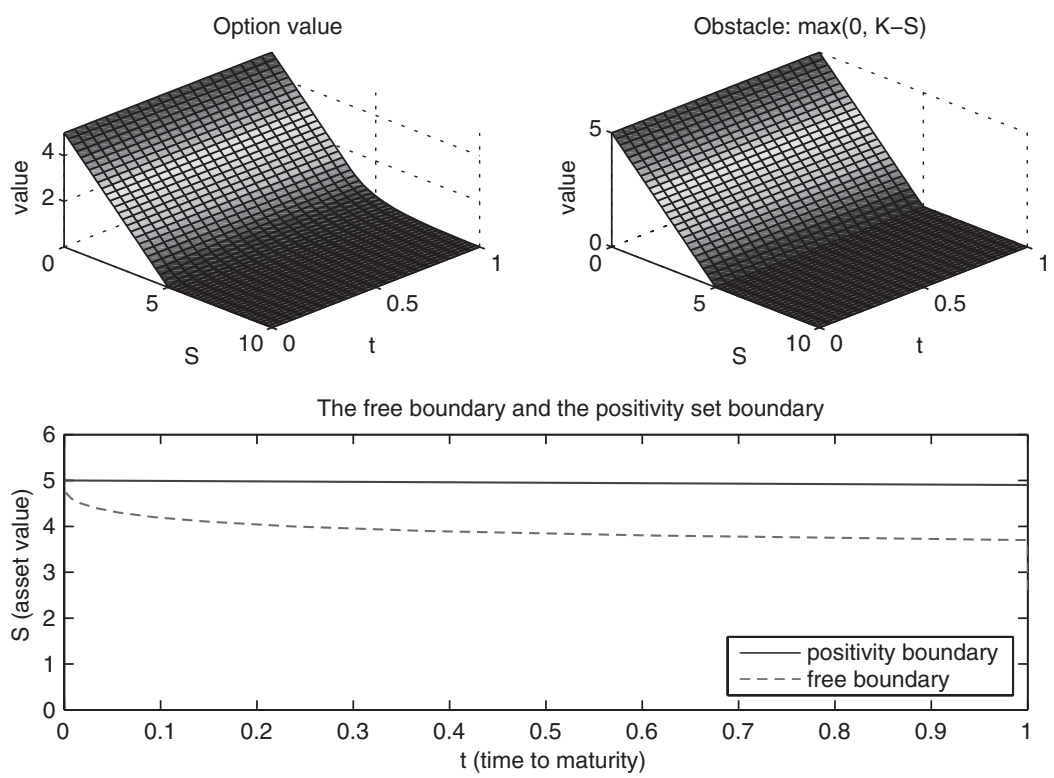

FIGURE 1 . Free boundary and the positivity set boundary for the obstacle $\phi=$ $\max \{0, K-s\}$. 

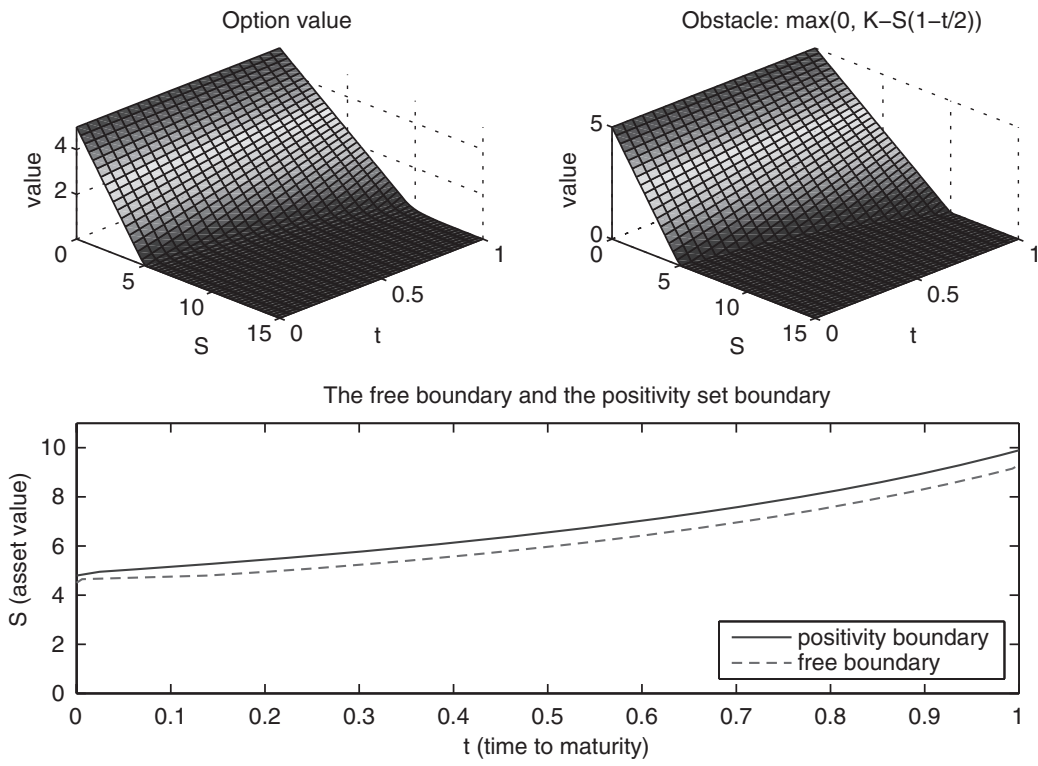

FIGURE 2. Free boundary and the positivity set boundary for the obstacle $\phi=\max \{0, K-s(1-t / 2)\}$.
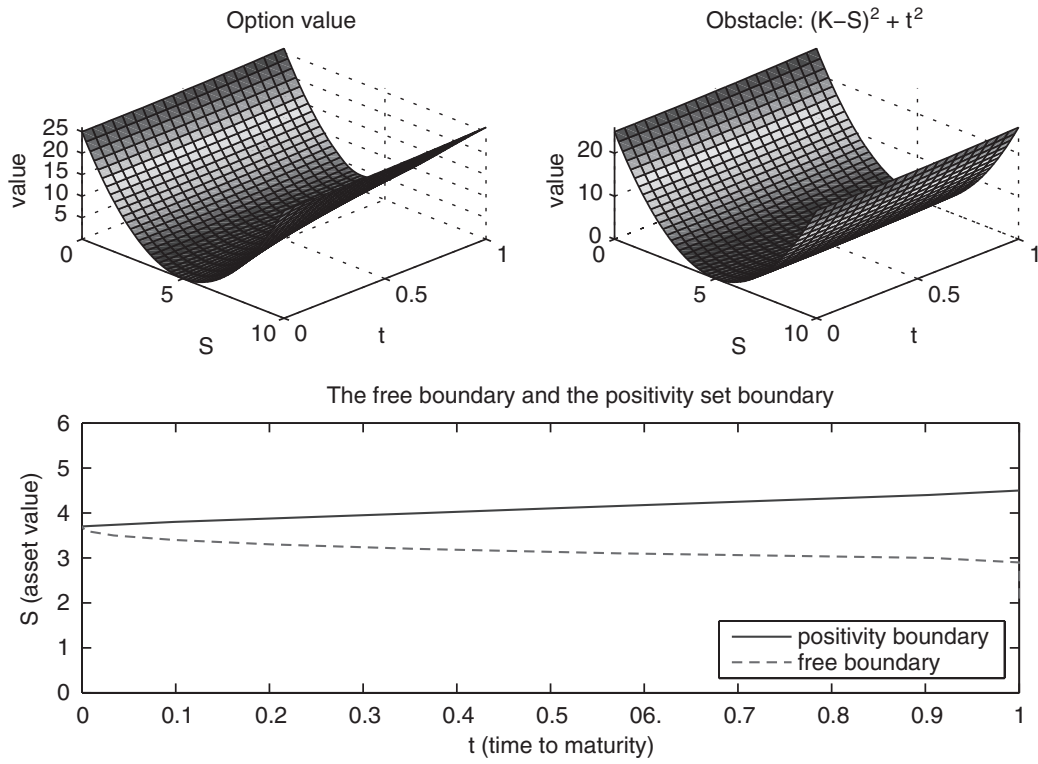

FIGURE 3. Free boundary and the positivity set boundary for the obstacle $\phi=(K-s)^{2}+t^{2}$. 


\section{REFERENCES}

1. Friedman, A., Partial Differential Equations of Parabolic Type, Prentice-Hall, 1964.

2. Friedman, A., Variational Principles and Free-Boundary Problems, Wiley, 1982.

3. Lieberman, G. M., Regularized distance and its applications, Pacific J. Math. 117(2) (1985), 329-352.

4. Merton, R. C., Theory of rational option pricing, Bell J. Econom. Management Sci. 4 (1973), $141-183$.

5. Caffarelli, L., Kenig, C., Gradient estimates for variable coefficient parabolic equations and singular perturbation problems, Amer. J. Math. 120(2) (1998), 391-429.

6. Ladyzhenskaya, O. A., Solonnikov, V. A., and Uraltseva, N. N., Linear and Quasi-Linear Equations of Parabolic Type, Transl. Math. Monogr. 23 (1968).

7. Musiela, M., Rutkowski, M., Martingale Methods in Financial Modelling, 2. ed., Springer, Berlin, Heidelberg [u.a.], 2005.

8. Jaillet, P., Lamberton, D., and Lapeyre, B., Variational inequalities and the pricing on American options, Acta Appl. Math. 21 (1990), 263-289.

MATEMATIKINSTITUTIONEN

KTH

SE-10044 STOCKHOLM

SWEDEN

E-mail: teitur@math.kth.se 